\title{
Soft computing methods for geoidal height transformation
}

\author{
O. Akyilmaz, M. T. Özlüdemir, T. Ayan, and R. N. Çelik \\ Istanbul Technical University, Faculty of Civil Engineering, Division of Geodesy, \\ 34469 Maslak, Istanbul, Turkey
}

(Received June 18, 2008; Revised December 2, 2008; Accepted January 6, 2009; Online published August 31, 2009)

\begin{abstract}
Soft computing techniques, such as fuzzy logic and artificial neural network (ANN) approaches, have enabled researchers to create precise models for use in many scientific and engineering applications. Applications that can be employed in geodetic studies include the estimation of earth rotation parameters and the determination of mean sea level changes. Another important field of geodesy in which these computing techniques can be applied is geoidal height transformation. We report here our use of a conventional polynomial model, the Adaptive Network-based Fuzzy (or in some publications, Adaptive Neuro-Fuzzy) Inference System (ANFIS), an ANN and a modified ANN approach to approximate geoid heights. These approximation models have been tested on a number of test points. The results obtained through the transformation processes from ellipsoidal heights into local levelling heights have also been compared.
\end{abstract}

Key words: Fuzzy inference systems, neural network, geoid undulation.

\section{Introduction}

Height determination is one of the important tasks of surveying and geodesy. Satellite-based positioning techniques provide ellipsoidal heights. However, most users desire heights in a natural system rather than purely geometric ellipsoidal heights. The most common natural system is orthometric datum, which has a physical context and depends on Earth's gravity field and, as such, refers to the geoid. The relation between ellipsoidal heights and orthometric heights can be established by geoid determination.

The most common method currently employed for precise geoid determination is the gravimetric method. However, the application of this technique is mainly dependent on the availability of high-resolution gravity data. In addition, the terrestrial gravity networks usually follow the road network and do not cover the high mountains where the geoid heights change significantly. In the absence of adequate gravity data, the geoid can be modelled using other geometric methods, such as the astro-geodetic method or geoid height from GPS in conjunction with spirit levelling (Kuhar et al., 2001). However, in most of the GPS applications, users need to transform ellipsoidal heights into orthometric heights in order to make them compatible with the existing orthometric heights on the local vertical datum (Engelis et al., 1985; Seeber, 2003).

The orthometric height is the distance of a point above the geoid measured along the plumb line through the point. The ellipsoidal height is calculated along the ellipsoidal normal, from the surface of any reference ellipsoid to the point of interest. The geoid height or geoid-ellipsoid separation is calculated along the ellipsoidal normal, from the

Copyright (c) The Society of Geomagnetism and Earth, Planetary and Space Sciences (SGEPSS); The Seismological Society of Japan; The Volcanological Society of Japan; The Geodetic Society of Japan; The Japanese Society for Planetary Sciences; TERRAPUB surface of any reference ellipsoid to the geoid. Therefore, an essential requirement of any transformation of ellipsoidal heights to orthometric heights is that the geoid height must refer to the same reference ellipsoid (Featherstone, 2001).

From the above definitions, the geoid height at each point can be given as follows,

$$
\xi \approx h-H
$$

where $\xi$ is either the geoidal undulation or the height anomaly, $h$ is the ellipsoidal height and $H$ is the orthometric height or the normal height. The approximate equality in the equation results from neglecting the departure of the plumbline from the ellipsoidal normal, which is called the deflection of the vertical. There is also torsion in the plumbline, but the deflection of the vertical is usually the dominant effect of the approximation in Eq. (1). The approximation error can be estimated by multiplying the orthometric height by the cosine of the deflection of the vertical at the point of interest. However, the maximum error of this approximation is $1-2 \mathrm{~mm}$, which is considerably smaller than the accuracy with which GPS-derived ellipsoidal and orthometric heights can currently be determined. Therefore, the approximation in Eq. (1) remains valid for the transformation of heights (Akyilmaz et al., 2003).

The determination of the geoid is in fact the interpolation of the known geoid heights at the control points that are located and distributed properly on the ground. However, the accuracy of the geoid depends on the accuracy of the input data, i.e. the accuracy and the density of the known geoid heights, rather than the method used for interpolation. Different methods have been widely employed for this purpose to date, including the least squares collocation, multi-parameter polynomial fitting (Ayan et al., 2001) and multi-quadratic or weighted linear interpolations (Yanalak 


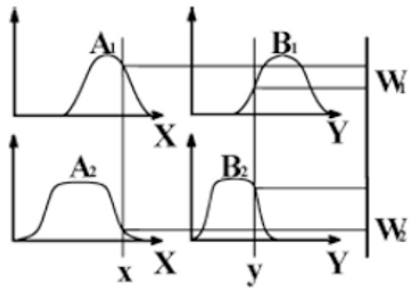

$\mathbf{f}_{1}=\mathbf{p}_{1} \mathbf{x}+\mathbf{q}_{1} \mathbf{y}+\mathbf{r}_{1}$

$\mathbf{f}_{2}=\mathbf{p}_{2} \mathbf{x}+\mathbf{q}_{2} \mathbf{y}+\mathbf{r}_{2}$

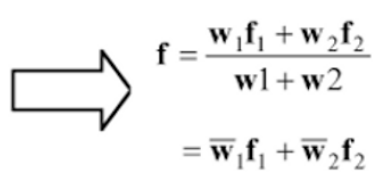

(a)

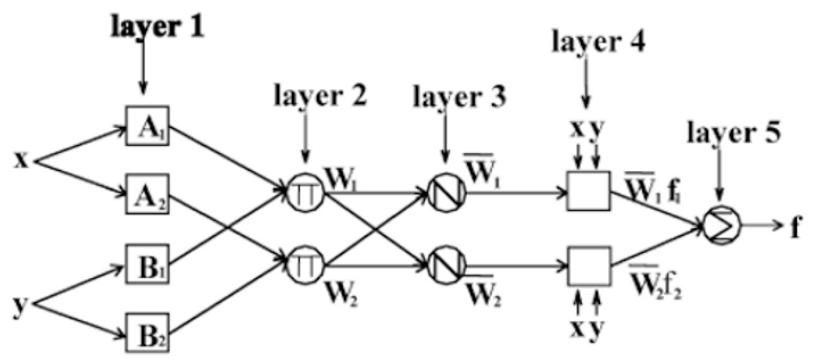

(b)

Fig. 1. (a) Type 3 fuzzy reasoning. (b) Equivalent ANFIS (Type 3 ANFIS).

and Baykal, 2001) by different auto-covariance functions. All of these methods have been evaluated only from the mathematical point of view and have often neglected the physical aspects of the geoid. The main advantage of the soft computing techniques is that the physical characteristics of the geoid are also taken into consideration to some extent-either explicitly or implicitly.

The study reported here focussed on the geoidal height transformation applications of two soft computing methods, namely the Adaptive Network-based Fuzzy (or in some publications, Adaptive Neuro-Fuzzy) Inference System (ANFIS) and Artificial Neural Networks (ANN).

Akyilmaz et al. (2003) and Akyilmaz and Kutterer (2004) provide an end-to-end description of the ANFIS approach, and the ANN methodology applied in this study is described by Akyilmaz et al. (2004). We have modelled the geoid in Izmir (Turkey) by applying these methodologies. Another mathematical method employed for geoid determination is the fifth-order multi-parameter polynomial approximation (Akyilmaz et al., 2003). In addition to employing the soft computing methods and the polynomial approximation, we also applied a least-squares collocation procedure in order to recover the remaining fine structure of the local geoid. In the following sections of this article, we report on the techniques and computing methodology used in our study and compare the soft computing methodologies.

\section{Adaptive Network-based Fuzzy Inference Sys- tem}

The ANFIS has emerged as an extension of fuzzy logic and fuzzy set theory introduced by Zadeh (1965). The benefit of ANFIS is that the parameters of the fuzzy sets defined in the inference model are optimised using proper mathematical algorithms when there is input-output data pairs available on the problem in question. The basic principle of ANFIS can be described as follows: a fuzzy inference sys-

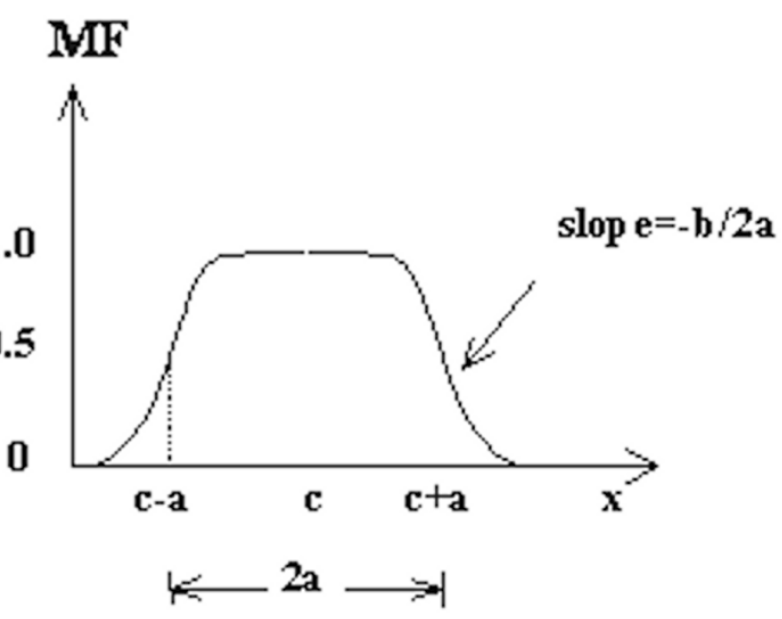

Fig. 2. Meanings of the parameters in the generalised bell membership function.

tem is typically designed by defining linguistic input and output variables as well as an inference rule base. However, the resulting system is just an initial guess for an adequate model. Hence, its premise and consequent parameters have to be tuned based on the given data in order to optimise system performance. In ANFIS, this step is based on a supervised learning algorithm (Akyilmaz et al., 2003).

An example of an ANFIS with two fuzzy rules are in the following form.

Rule 1: If $x \in A_{1}$ and $y \in B_{1}$; then $f_{1}=p_{1} x+q_{1} y+r_{1}$. Rule 2: If $x \in A_{2}$ and $y \in B_{2}$; then $f_{2}=p_{2} x+q_{2} y+r_{2}$.

The associated fuzzy reasoning is illustrated in Fig. 1(a), and the corresponding equivalent ANFIS architecture is shown in Fig. 1(b). 
The parameters of the input fuzzy sets (also named as membership functions) $A_{i}, B_{j}(i=1,2 j=1,2)$ are called the premise parameters, whereas the parameters of output functions $f_{i}(i=1,2)$, i.e. $p_{i}, q_{i}$ and $r_{i}$, are called consequent parameters (Takagi and Sugeno, 1985). The input membership function $\mu_{A_{i}}(x)$ is usually chosen to be bell-shaped (Eq. (2)), with the maximum value equal to 1 and the minimum value equal to 0 (such as the generalised bell function; Fig. 2),

$$
\mu_{A_{i}}(x)=\frac{1}{1+\left[\left(\frac{x-c_{i}}{a_{i}}\right)^{2}\right]^{b_{i}}}
$$

or the Gaussian function

$$
\mu_{A_{i}}(x)=\exp \left[-\left(\frac{x-c_{i}}{a_{i}}\right)^{2}\right]
$$

where $\left\{a_{i}, b_{i}, c_{i}\right\}$ (or $\left\{a_{i}, c_{i}\right\}$ in the case of the Gaussian function) is the parameter set. As the values of these parameters change, the bell-shaped functions vary accordingly.

The interested reader desiring more information on the set up of ANFIS and the tuning up of the parameters are referred to Jang et al. (1997) and Akyilmaz and Kutterer (2004).

The procedure for the application of ANFIS to geoidal height transformation can be summarized step-by-step as follows:

1) Calculation of the observed geoid heights using Eq. (1). Beforehand, the outliers have to be removed from the data set by statiscal testing of the both GPS and levelling of the derived heights. This is performed during the adjustment of the observations.

2) Separation of the entire data into two independent groups as training and test data sets, respectively. Training data are the reference points which will be used to estimate the ANFIS model parameters, whereas the test data set will be used to validate the estimated model parameters. The test data set should be distributed as homogenously as possible because the extrapolation is usually problematic. In practice, usually at least $20 \%$ of all data points can be selected as the test data.

3) Selection of the input variables. The input variables may be selected as the geographic coordinates of the points or the geographic coordinates and the ellipsoidal heights of the points, as adopted in this study.

4) Assignment of the input membership functions to each input variable. Different types and numbers of membership functions are possible. However, for geodetic applications, membership functions of the Gaussian type are the most appropriate ones. The optimum number of membership functions for each input variable is usually determined by trials regarding the performances of the relevant ANFIS models. The output membership functions are the first order polynomials of the input variables. The number of the output membership functions depend on the number of fuzzy rules. which is the number of all combinations of the input membership functions.

5) Training of the network. After the ANFIS structure (i.e. the number and the type of the input membership functions were determined) has been fixed, the parameters of both the input membership functions and the output membership functions have to be adjusted iteratively. There are several mathematical methods that can be used for the training. The hybrid training algorithm proposed by Jang (1993) is preferred because of its rapid convergence to the global optimum solution.

6) Validation of the estimated ANFIS parameters. Once the parameters of the ANFIS are estimated after the training step, they are used to compute the geoid heights at the test data points. The RMS error of the computed geoid heights for the training data and the test data, respectively, should be compatible_-in other words, they should be close to each other. If not, the composed set up of the ANFIS is not adequate, and the number of membership functions should be changed (i.e., go to step 3). The trials should be repeated until a compromise between the RMS errors of the training and test data points are achieved.

7) End.

\section{Multilayer Feed-forward Networks}

Commonly used artificial neural networks (ANN) are multilayer feed-forward (MLFF) networks. Neural networks typically consist of many simple neurons located on different layers that operate in cooperation with the neurons on the other layers in order to achieve a good mapping of input-to-output signals. The expression feed-forward emphasises that the flow of the computation is from input towards the output. There are three different types of layers in the concept of neural networks: the input layer (the one to which external stimuli are applied to), the output layer (the layer that results in output) and hidden layers (intermediate computational layers between input and output). Theoretically, there is no limitation given for the number of hidden layers in a network configuration. The absence of such limitations, however, has a great effect in the computation time as well as on the number of neurons in hidden layers. Therefore, a compromise has to be found in order to achieve an optimal network configuration with an acceptable convergence time and quantitative precision.

Figure 3 provides a sample configuration of a MLFF network with one input, one output and one hidden layer. Note that the network consists of five inputs and one output.

When a network configuration is fixed, the parameters of the network, i.e. the weights which link the neurons in consecutive layers, have to be calculated so that a chosen function of the difference between the actual (desired) output and the output performed by the network is at a minimum. This function is usually called the cost function or performance index. The most commonly used cost function is the sum of the squares of the residuals:

$$
E=\sum\left(y_{i}(k)-y_{i}^{\prime}(k)\right)^{2}
$$

where $y_{i}(k), y_{i}^{\prime}(k)$ and $E$ are the actual output, network output and the corresponding cost function, respectively. 


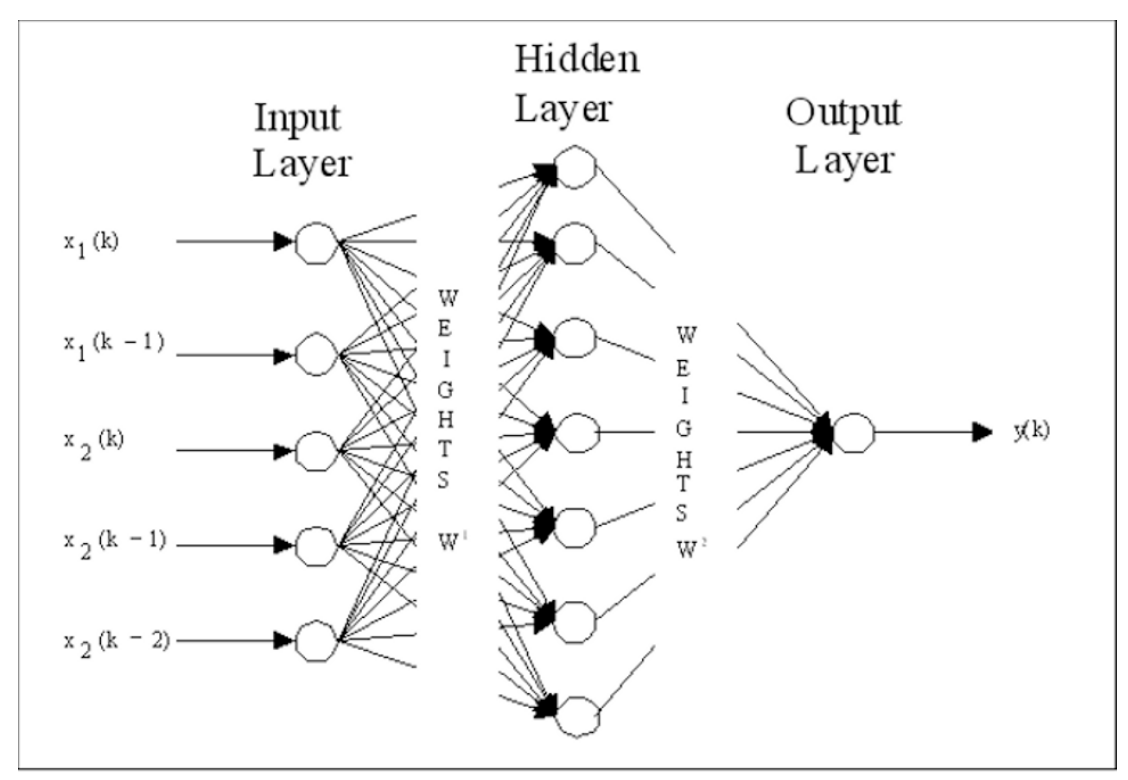

Fig. 3. A schematic representation of a multilayer feed-forward (MLFF) neural network with one hidden layer.

There is a wide spectrum of different mathematical optimisation tools which are based on the iterative least-squares estimation of the network parameters, such as the steepest gradient descent, the Levenberg-Marquardt method, the Gauss-Newton method, among others. These are not discussed in detail here but full information can be found in standard textbooks, such as those of Bishop (1995) and Haykin (1994). The procedure for the optimisation of the network parameters is usually called learning or training in neural computing literature.

Hu et al. (2004) applied a two-step approach consisting of the combination of surface function fitting followed by the ANN approximation. In our study we have applied both the conventional ANN approach and the aforementioned hybrid ANN approach introduced by $\mathrm{Hu}$ et al. (2004) to the same data to convert the ellipsoidal heights obtained through GPS positioning into the orthometric heights.

The procedure we followed for the application of ANN to geoidal height transformation can be summarized stepby-step as follows:

1) Calculation of the observed geoid heights using Eq. (1). Beforehand, the outliers have to be removed from the data set by statiscal testing of the both GPS and levelling derived heights.

2) Separation of the entire data into two independent groups as training and test data sets, similar to the case in the ANFIS method.

3) Selection of the input variables. The input variables might be selected as the geographic coordinates of the points or as the geographic coordinates and the ellipsoidal heights of the points, as adopted in this study.

4) Setting up the ANN architecture. This step includes the determination of the number of hidden layers and the number of neurons at each hidden layer. The number of input and output layers is known prior to this step as they are equal to the number of input and output variables, respectively. As a rule, one tries to achieve a reasonable network architecture with as few as possible hidden layers and neurons. This is usually related to the complexity of the process in question. However, for geoidal height transformation, one or two hidden layers are sufficient.

5) Initialization of the training parameters. Before the training has started, some initial parameters have to be defined. These are the initial values of the weights between the neurons in successive layers (see Fig. 3), the "learning rate" and the expected performance of the ANN (also called the "goal" of the ANN) in terms of average RMS error.

The initial values for the weights are usually assigned by a random number generator. Most of the ANN softwares use random number generators to determine the initial weights. However, one can assign the value of 1 to all the weights as the initial values.

The learning rate is in fact the parameter related to the step-size of the iterative adjustment of the weights between the neurons. It is usually selected to be a constant value between 0.01 and 0.10 . A high value of the learning rate may yield overtraining, whereas a low value may yield a slow convergence. Therefore, a compromise has to be found for the learning rate. For the numerical example in this study, the value of 0.08 is used.

The goal of the ANN can be defined most simply as the average RMS error of the ellipsoidal heights, which are readily available from the GPS network adjustment. The contribution of the spirit levelling heights to the RMS error of the geoidal undulations can be neglected as they are much more accurate that to the accuracy of the ellipsoidal heights.

6) Training of the ANN. Once the ANN architecture and the training parameters are fixed, the parameters of the ANN, i.e. the weights between the neurons in successive layers (see Fig. 3), have to be adjusted itera- 
tively. There are numerious methods for updating this parameter in ANN applications. The most commonly used one is the so-called "error back-propagation" algorithm, which is also known as the "steepest gradient descent" algorithm in numerical analysis. In the case of ANNs with a single output (e.g. this study), the Levenberg-Marquardt algorithm can be adopted as it converges more rapidly than the error backpropagation algorithm. Note that all of these training algorithms are gradient-based methods with slight differences from each other. The training is terminated when the predefined goal (i.e. the average RMS error) is achieved.

7) Validation of the ANN parameters. After the training is terminated, the final estimate of the ANN parameters, i.e. the weights, are obtained. These weights are then applied to the test data. The RMS for the test data and that for the training data have to be compatible with each other. If not, the ANN architecture, i.e. the number of the neurons in the hidden layer, has to be altered (go to step 4), and the process should be repeated until a compromise between the training and test RMS errors are achieved.

8) End.

\section{Numerical Applications}

The data used in this study were collected within the Izmir Geodetic Reference System-2001 project (Ayan et $a l ., 2001)$. Using these data, we have applied the ANFIS, ANN and fifth-order polynomial approximation methods for geoid determination.

As shown in Fig. 4, the region of Izmir covers an area of approximately $d_{\varphi}=0.30^{\circ}$ and $d_{\lambda}=0.56^{\circ}$. The geoid heights in the area of interest vary from 37.6 to $38.7 \mathrm{~m}$. Although the point density is high in the region, the average accuracy, i.e. the average RMS error, of the ellipsoidal heights after the adjustment of the network was found to be $\pm 3.5 \mathrm{~cm}$. The accuracy of the ellipsoidal heights varies from $\pm 2.8 \mathrm{~cm}$ to $\pm 5.0 \mathrm{~cm}$. The region fortunately does not contain very high mountains, and the levelling measurements could therefore also be carried out to the points on relatively high hills. Since the soft computing methods cannot handle blunders, adjusted GPS baseline vectors were statistically tested and blundered observations removed from the data set by an iterative procedure (Baarda, 1968). Hence, the data set do not include any outliers. Additionally, in terms of Fig. 4, the point distribution is not very optimal. The number of the points with known geoid heights is 310 . Of these, 75 uniformly distributed points (approximately $25 \%$ of the data set), marked with squares in Fig. 4, were randomly selected to form a test set. The remaining 235 points, marked with filled circles in Fig. 4, were used for training ANFIS and ANN networks.

The first step in our study was to apply ANFIS to transform the geoidal height. For these computations, we used the geographic coordinates $\varphi, \lambda$, and $h$ as input variables and the geoid height $(\xi)$ as the output of the system. The computations were repeated by using different number of input membership functions of Gaussian type. The different ANFIS configurations that resulted from these compu-

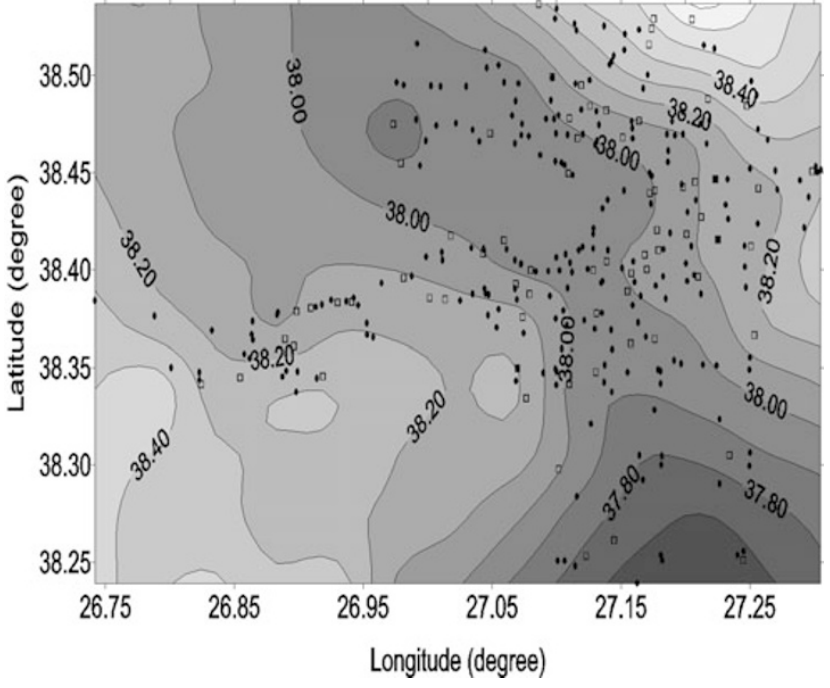

Fig. 4. Local geoid model in Izmir region.

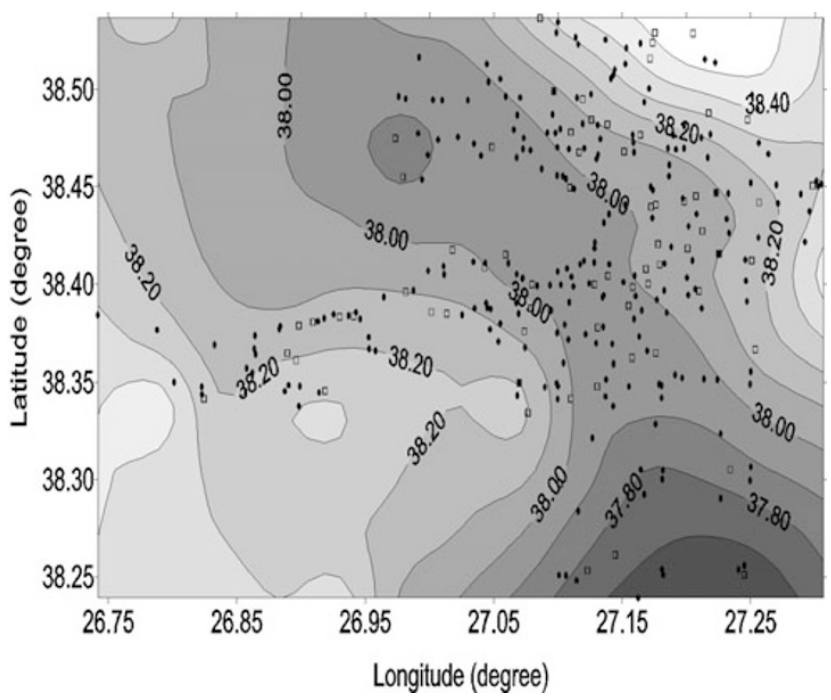

Fig. 5. Geoid heights obtained from the ANFIS-only method.

tations were then validated using the test data. After fewer than ten trials, the optimal ANFIS configuration was determined to be five, three and one fuzzy sets for the representation of the input variables, respectively. Figures 5 and 6 show the estimated geoid heights and the distribution of the corresponding errors in the study area, respectively, that were obtained by applying the ANFIS method.

The second step was the application of ANN analogy. In this application, after hundreds of trials were conducted by changing either the learning rate, training algorithm, number of layers, number of neurons in the layers, and the network type, we found that the optimal ANN configuration was the feed-forward network with two hidden layers, each of which contains 13 neurons. The training accuracy of the study set was also determined by trials, and the final accuracies presented here are the best ones obtained after these trials. Moreover, these final accuracies are consistent with the accuracies of the adjusted ellipsoidal heights, which are dominant for the accuracy of the geoidal undulations compared to levelling heights. This was an expected result. 


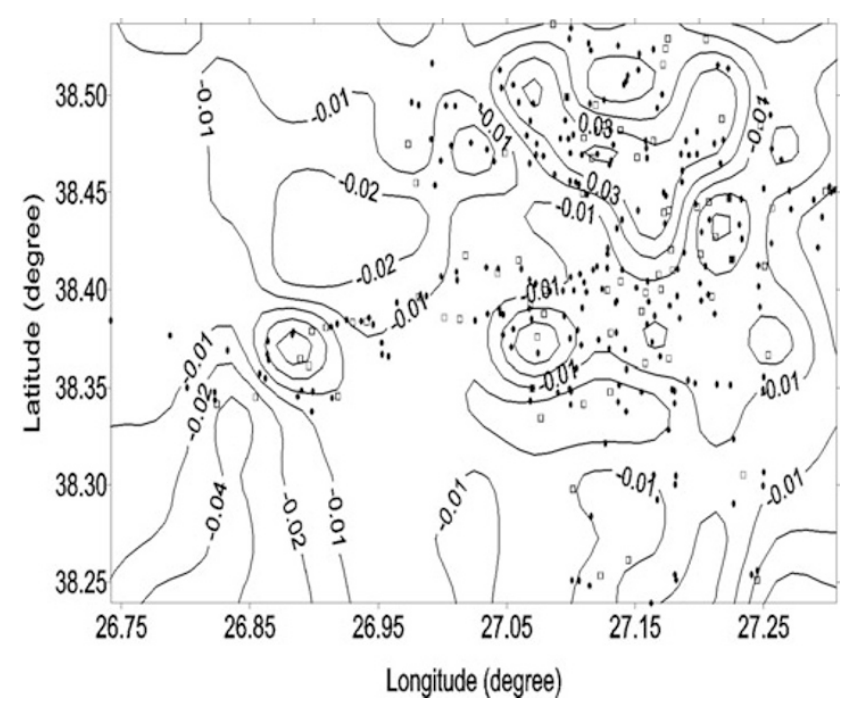

Fig. 6. Differences (residuals) between observed geoid heights and geoid heights computed from the ANFIS-only method.

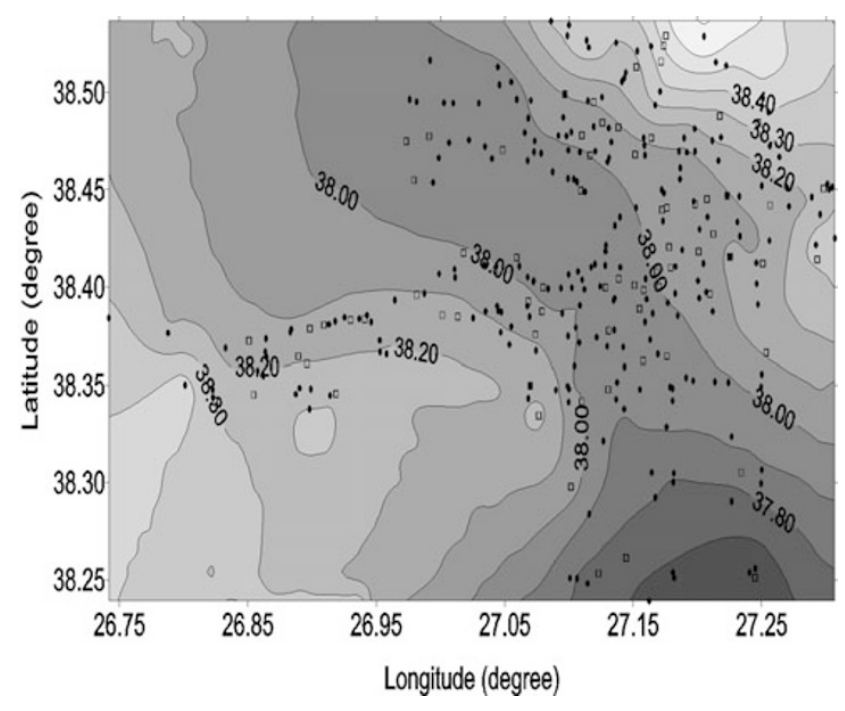

Fig. 7. Geoid heights obtained from the ANN-only method.

In the above-mentioned applications, the input components were taken as the ellipsoidal geographic coordinates $\varphi, \lambda, h$; and the output was the geoid height ( $\xi$ ) (Akyilmaz et al., 2003). In comparison with the ANFIS approximation, it takes a relatively long time to carry out the procedure in the ANN analogy. On the other hand, since the initial weights are randomly drawn values, one can achieve different results for the same ANN configuration after implementing different training procedures. Figures 7 and 8 illustrate the estimated geoid heights and the corresponding errors in the study area, respectively obtained from the ANN method.

The same data have also been used for the fifth-order polynomial approximation. Table 1 summarises the performances of the ANFIS and ANN methodologies as well as that of the fifth-order polynomial model. Note that the fifthorder polynomial approximation using least squares adjustment has been performed twice; first, using all 310 points to determine the coefficients of the polynomial, and sec-

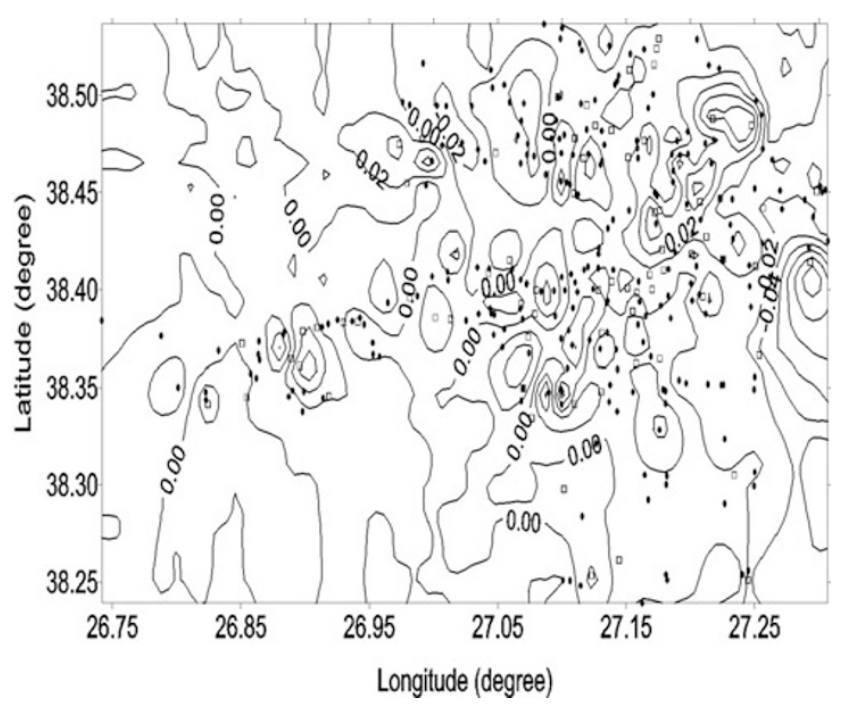

Fig. 8. Differences (residuals) between observed geoid heights and geoid heights computed from the ANN-only method.

ond, using 235 points only to determine the coefficients. The coefficients of the polynomial approximation were also tested for significance; and non-significant ones were then removed from the functional model, and the adjustment was repeated until all the coefficients were statistically significant. Polynomial approximations for higher degrees were also computed; however, there were no reasonable improvements in the solutions and to maintain the degree of freedom, the fifth order polynomial was adopted for this study.

The computations were carried out in twofold. In the first phase, only the proposed methods were directly applied; in the second phase, the computed geoidal undulations were assumed to be the deterministic part (trend surface) of a least squares collocation (LSC) problem. The unmodelled fine structure of the local geoid surface is then further estimated by the LSC. Several auto-covariance functions were applied for this computation, with Hirvonen's auto-covariance function with a correlation distance of $1 \mathrm{~km}$ found to be the most appropriate one of all those tested (Ayan, 1976; Moritz, 1978; Jasecki, 1983).

As seen from Table 1, the quality measures obtained for training points by the ANN methodology are slightly better than those obtained by ANFIS approach and the fifth-order polynomial model. The latter gives the worst results compared with the other methodologies. A comparison of the quality measures for the test points leads to the conclusion that the ANFIS approximation yields better results than the others. A review of the results after the application of LSC shows that there is no improvement for the case of ANN and ANFIS. In contrast, there is a significant improvement in the results of the polynomial approximation. This improvement is due to the fact that the soft computing methods, such as ANN and ANFIS, tend to describe the surface by piecewise (either linear or non-linear) functions, and the entire study area is covered by these locally best fitting piecewise functions. Therefore, a further LSC is not essential while the user has found the optimal configuration as well as the tuning parameters for the soft computing methods. However, in the case of the polynomial approximation, finer de- 
Table 1. Comparison between ANFIS, ANN and the fifth-order approximations (with and without LSC applied).

\begin{tabular}{|c|c|c|c|c|c|c|}
\hline Approximation type & Minimum [m] & Maximum [m] & Mean $[\mathrm{m}]$ & $\begin{array}{c}\text { Mean absolute } \\
\text { deviation }[\mathrm{m}]\end{array}$ & $\begin{array}{c}\text { Standard } \\
\text { deviation }[\mathrm{m}]\end{array}$ & $\begin{array}{c}\text { Correlation } \\
\text { coefficient }\end{array}$ \\
\hline 235 points ANFIS & -0.111 & 0.098 & 0.000 & 0.021 & 0.030 & 0.98335 \\
\hline 235 points $\mathrm{ANN}$ & -0.093 & 0.106 & 0.001 & 0.022 & 0.030 & 0.98403 \\
\hline 235 points fifth order & -0.135 & 0.126 & 0.000 & 0.033 & 0.043 & 0.97362 \\
\hline 310 points fifth order & -0.156 & 0.122 & 0.000 & 0.033 & 0.044 & 0.96527 \\
\hline 75 points ANFIS & -0.090 & 0.141 & 0.000 & 0.027 & 0.037 & 0.97222 \\
\hline 75 points ANFIS + LSC & -0.090 & 0.143 & 0.000 & 0.027 & 0.038 & 0.97366 \\
\hline 75 points $\mathrm{ANN}$ & -0.158 & 0.132 & 0.002 & 0.029 & 0.040 & 0.97981 \\
\hline 75 points $\mathrm{ANN}+\mathrm{LSC}$ & -0.152 & 0.136 & 0.001 & 0.028 & 0.041 & 0.97311 \\
\hline 75 points fifth order & -0.122 & 0.105 & 0.000 & 0.035 & 0.045 & 0.96462 \\
\hline 75 points fifth order + LSC & -0.131 & 0.084 & 0.000 & 0.028 & 0.039 & 0.96990 \\
\hline
\end{tabular}

Table 2. Comparison between the hybrid ANFIS and ANN results (with and without LSC applied).

\begin{tabular}{|c|c|c|c|c|c|c|}
\hline Approximation type & Minimum [m] & Maximum [m] & Mean $[\mathrm{m}]$ & $\begin{array}{l}\text { Mean absolute } \\
\text { deviation }[\mathrm{m}]\end{array}$ & $\begin{array}{c}\text { Standard } \\
\text { deviation }[\mathrm{m}]\end{array}$ & $\begin{array}{l}\text { Correlation } \\
\text { coefficient }\end{array}$ \\
\hline 235 points ANFIS & -0.095 & 0.113 & 0.000 & 0.027 & 0.036 & 0.97657 \\
\hline 235 points ANN & -0.106 & 0.128 & 0.002 & 0.025 & 0.034 & 0.97841 \\
\hline 75 points ANFIS & -0.110 & 0.143 & 0.002 & 0.030 & 0.040 & 0.96743 \\
\hline 75 points ANFIS + LSC & -0.106 & 0.140 & 0.002 & 0.028 & 0.039 & 0.97427 \\
\hline 75 points ANN & -0.107 & 0.126 & 0.002 & 0.033 & 0.043 & 0.96561 \\
\hline 75 points $\mathrm{ANN}+\mathrm{LSC}$ & -0.105 & 0.134 & 0.000 & 0.031 & 0.042 & 0.96772 \\
\hline
\end{tabular}

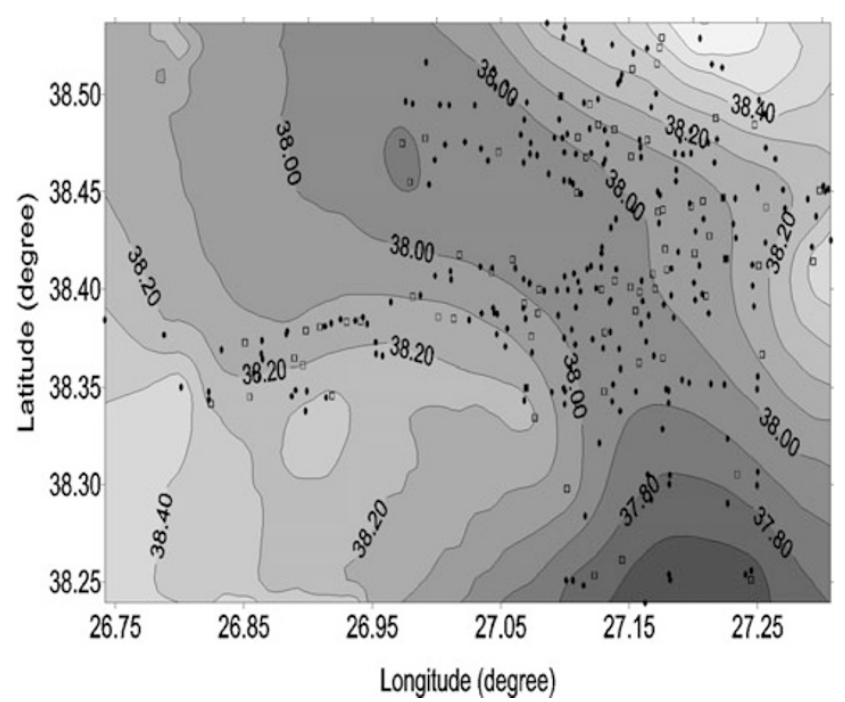

Fig. 9. Geoid heights obtained from the hybrid ANFIS method.

tails of the geoid can be extracted by applying a LSC. This is one of the important findings of the present study.

In the second phase of the computations, the ANFIS and ANN approximations, modified with respect to the methodology proposed by $\mathrm{Hu}$ et al. (2004), were applied. First, the 235 points were used as the training group and the fifthorder polynomial coefficients were estimated by the leastsquares estimation. Then, using these coefficients, we computed approximate geoid height values $\left(\xi_{0}\right)$. Deviations from observed (the values computed by taking the difference between the adjusted ellipsoidal heights and levelling heights) geoid height values $(\Delta \xi)$ were then calculated and used as the output where $\varphi, \lambda$ and the $\xi_{0}$ were used as the

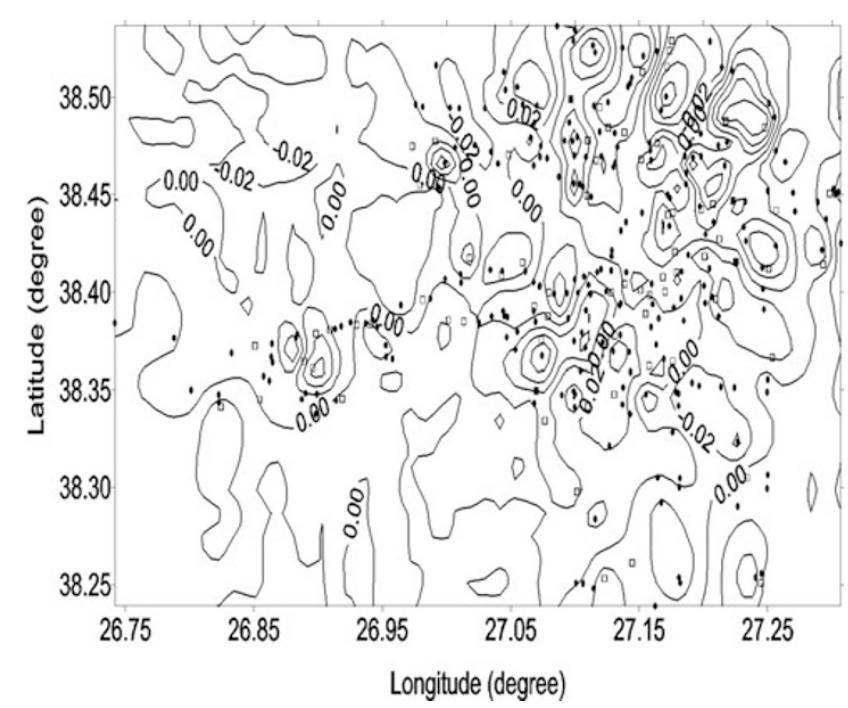

Fig. 10. Differences (residuals) between observed geoid heights and geoid heights computed from the hybrid ANFIS method.

input to feed both ANFIS and ANN (Hu et al., 2004). After fewer than ten trials, the optimal ANFIS configuration was found to be two fuzzy sets for each input variable, yielding eight fuzzy if-then rules. After hundreds of trials, the ideal ANN configuration was again found to be a two-layerfeed-forward network containing 13 neurons in each hidden layer. For both methods, 75 test points were used to check the performance of the models. Table 2 summarises the results of the mentioned methodologies. Figures 9 and 10 show the geoid heights estimated using the hybrid ANFIS method and the distribution of the corresponding errors in the study area, respectively. Similarly, Figs. 11 and 12 


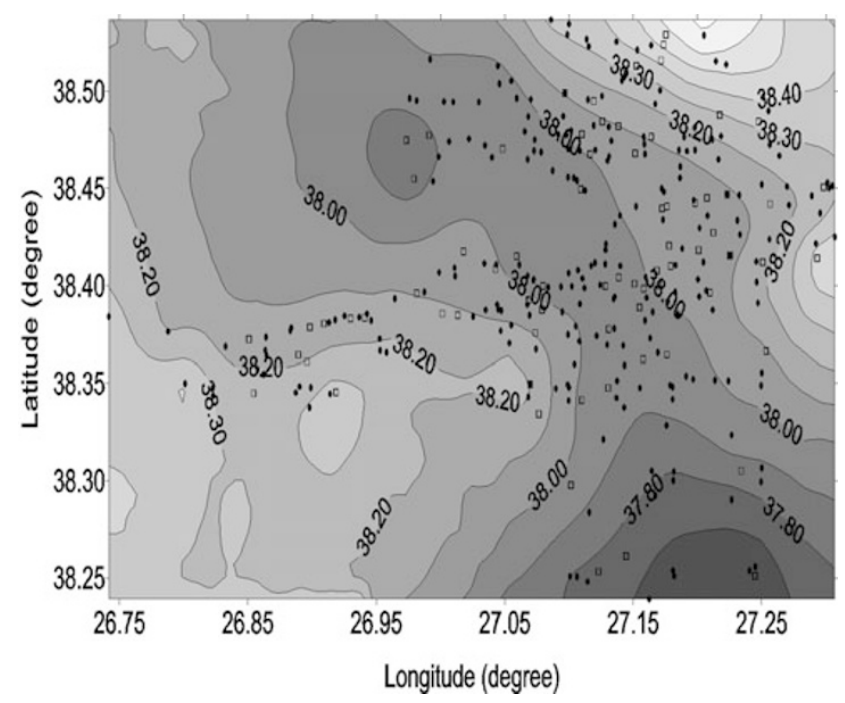

Fig. 11. Geoid heights obtained from the hybrid ANN method.

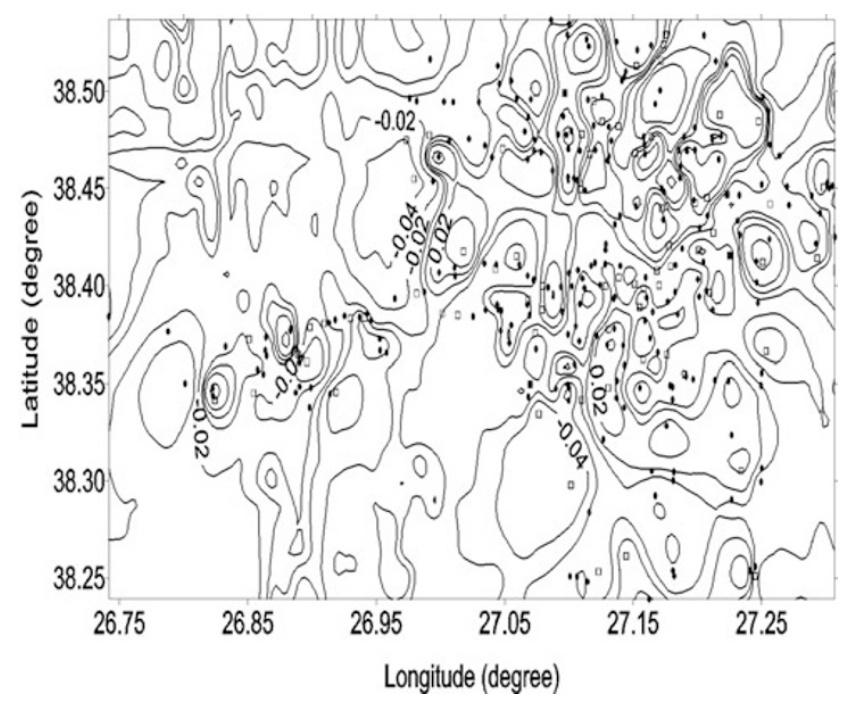

Fig. 12. Differences (residuals) between observed geoid heights and geoid heights computed from the hybrid ANN method.

present the results obtained from the hybrid ANN approach. A comparison of the results given in Table 1 shows that the hybrid method yields the worse results, which are due to the computed input observation $\xi_{0}$ contributing to the error budget of the ANN and ANFIS models. The best accuracies obtained for the ANFIS and ANN training sets were found to be $0.036 \mathrm{~m}$ and $0.034 \mathrm{~m}$, respectively, in terms of standard deviations. Changing the accuracy of the training sets to values equal or lower than $0.030 \mathrm{~m}$ yields worse accuracies for the test set.

From the results given in Table 2, it can be concluded that the quality measures obtained for the training points by the ANFIS methodology is slightly better than those obtained by the ANN approach. As seen, both the minimum and the maximum error values obtained by ANFIS are lower than those produced with the ANN analogy. When the quality measures for the test points are considered, the minimum and maximum values obtained by ANN are better. However, the standard deviation and correlation coefficient ob- tained by ANFIS are slightly better. We conclude that both methodologies yield identical results. Looking at the results after LSC, again there is no significant improvement both for ANN and ANFIS methods. This is due to the explanation mentioned above for the results given in Table 1.

\section{Conclusions}

This study deals with the application of Adaptive Network based Fuzzy Inference System (ANFIS) and Artificial Neural Networks (ANN) methods to geoidal height transformation. We also applied a conventional fifth-order polynomial approximation model to determine geoidal height transformation. The outputs obtained by these different approximation methodologies were then compared. A leastsquares collocation (LSC) procedure was also applied to the residuals obtained from the all methods.

When the results in Table 1 and Table 2 are compared, it is obvious that it is futile to conduct a pre-process by utilising a deterministic function which yields reduced values for the observed geoid heights established as the output for the patterns in both ANFIS and ANN methodologies. The results given by $\mathrm{Hu}$ et al. (2004) are quite precise, possibly because the study field covered a small and considerably smooth area and the height differences between the geodetic points were at a low level.

We found that ANFIS approximation yields better results than the classical ANN approximation and the conventional fifth-order polynomial model. However, the modified ANN analogy introduced by $\mathrm{Hu}$ et al. (2004) produces identical results with the ANFIS approximation.

A number of aspects of ANFIS have to be strictly controlled when using this application. One of these is that the number of parameters, including both premise and consequent, has to be less than the number of training data pairs. This avoids the overfitting phenomenon, which does not allow generalisation of the established fuzzy inference system.

Regardless of which method is used, the efficiency of the approximation is limited at least by the accuracy of the ellipsoidal heights obtained from the adjustment of the network as it has the dominant effect on the geoidal undulations or the height anomaly. This means that it is not possible to achieve an approximation of the accuracy that is better than the accuracy of the ellipsoidal heights. Given this limitation, the RMS error obtained by ANFIS is very close to the accuracy of adjusted ellipsoidal heights. Point distribution is also an important factor for the approximation quality. It should also be stated that the convergence of ANFIS is considerably faster than that of ANN. Moreover, the ANFIS approximation requires fewer parameters than ANN. For example, ANFIS modelling without polynomial fitting (Table 1) contains 78 parameters to be updated by the training runs. In the case of ANFIS combined with the pre-process (in Table 2), this is 44 . However, in the case of ANN, the number of parameters is 507 for both ANN approaches given in Tables 1 and 2 .

One important finding of our study is that the application of LSC to the results of ANN and ANFIS does not improve the results, which is not the case for the polynomial approximation. This lack of improvement is due to the fact that soft 
computing methods, such as ANN and ANFIS, tend to describe the surface in piecewise (either linear or non-linear) functions, and the entire study area is covered by these locally best fitting piecewise functions.

A study of Figs. 5-12 reveals that the ANFIS-only method provides a smoother view of the geoid heights as well as the corresponding errors in the study area. This result is due to the characteristics of the ANFIS method. In contrast, the hybrid-ANFIS results (Figs. 9 and 10), i.e. the application of LSC to the ANFIS residuals, has a corrupting effect on the smoothness and the distribution of the geoid height errors.

Acknowledgments. Dr. Takeshi Sagiya and the two anonymous referees are gratefully acknowledged for their constructive comments which greatly improved the quality of the manuscript.

\section{References}

Akyilmaz, O. and H. Kutterer, Prediction of Earth rotation parameters by fuzzy inference system, J. Geod., 78, 82-93, doi:10.1007/s00190-0040374-5, 2004.

Akyilmaz, O., T. Ayan, and M. T. Özlüdemir, Geoid surface approximation by using Adaptive Network based Fuzzy Inference Systems, $A V N, \mathbf{8 - 9}$, 308-315, 2003.

Akyilmaz, O., R. N. Çelik, N. Apaydin, and T. Ayan, GPS monitoring of the Fatih Sultan Mehmet suspension bridge by using assessment methods of neural networks. The International Archives of the Photogrammetry, Remote Sensing, Spatial Inf. Sci., 34, 702-707, 2004.

Ayan, T., Astrogeodetic Geoid Determination for the Area of Turkey, Ph.D. dissertation, Karlsruhe, Germany, 1976 (in German).

Ayan, T., R. Deniz, R. N. Çelik, H. H. Denli, M. T. Özlüdemir, S. Erol, B. Özöner, O. Akyilmaz, and C. Güney, Izmir geodetic reference system2001 (IzJRS-2001), Technical Report, Istanbul Technical University, Turkey, 2001 (in Turkish).

Baarda, W., A testing procedure for use in geodetic networks, Netherlands
Geodetic Commission, Publications in Geodesy, New Series, 2(5), Delft, The Netherlands, 1968.

Bishop, C. M., Neural networks for pattern recognition, Oxford University Press, 1995.

Engelis, T., R. H. Rapp, and Y. Bock, Measuring orthometric height differences with GPS and gravity data, Manuscripta Geodaetica, 10(3), 187-194, 1985.

Featherstone, W. E., Absolute and relative testing of gravimetric geoid models using Global Positioning System and orthometric height data, Comput. Geosci., 27, 807-814, doi:10.1016/S0098-3004(00)00169-2, 2001.

Haykin, S., Neural networks: a comprehensive foundation, Maxwell Macmillan Int., New York, 1994.

Hu, W., Y. Sha, and S. Kuang, New method for transforming Global Positioning System height into normal height based on Neural Network, J. Surv. Eng., 130(1), 36-39, 2004.

Jang, J. S., ANFIS: Adaptive-Network-Based Fuzzy Inference System, IEEE Trans. Systems Man Cybernetics, 23, 665-685, 1993.

Jang, J. S., C. Sun, and E. Mizutani, Neuro-Fuzzy and Soft Computing, Prentice-Hall, Englewood Cliffs, NJ, 1997.

Jasecki, M., The covariance function in the process of developing geodetic data, Geodesy Cartography, PWN, 32(4), 283-292, Warsaw, 1983.

Kuhar, M., B. Stopar, G. Turk, and T. Ambrožič, The use of artificial neural network in geoid surface approximation, $A V N$, 1, 22-27, 2001.

Moritz, H., Least-squares collocation, Rev. Geoph. Space Physics, 16(3), 1978.

Seeber, G., Satellite Geodesy, 2nd revision edition, Walter de Gruyter Inc., 2003.

Takagi, T. and M. Sugeno, Fuzzy identification of systems and its application to modelling and control, IEEE Trans. Systems Man Cybernetics, 15, 116-132, 1985.

Yanalak, M. and O. Baykal, Transformation of ellipsoidal heights to local levelling heights, ASCE J. Surv. Eng., 127(3), 90-103, 2001.

Zadeh, L. A., Fuzzy sets, Inf. Control, 8, 338-353, 1965

O. Akyilmaz (e-mail: akyilma2@itu.edu.tr), M. T. Özlüdemir, T. Ayan, and R. N. Çelik 